\title{
Myoclonus and ataxia occurring in a family
}

\author{
HAROLD JACOBS
}

From the Service of Neurology, Regina General and Grey Nuns' Hospitals, Saskatchewan, Canada

In 1914 J. Ramsay Hunt described the syndrome he called 'dyssynergia cerebellaris progressiva'. He regarded this condition with its well-defined cerebellar symptoms as an 'organic disease caused by degeneration of certain special structures of the cerebellar mechanism'. In 1921 at an annual meeting of the American Neurological Association, he presented a further six cases which combined the symptoms of dyssynergia cerebellaris progressiva with myoclonus and epilepsy. Only two of his patients were related. Both patients, twins, had in addition Friedreich's ataxia, and of these, one came to necropsy. Pathological studies on this 36-year-old male showed primary atrophy of the efferent dentate system of the cerebellum with "considerable diminution in number and extensive atrophy of the cells of the dentate nucleus with secondary atrophic changes in the superior cerebellar peduncles', as well as the typical spinal lesions of Friedreich's ataxia. Ramsay Hunt now called this syndrome 'dyssynergia cerebellaris myoclonica'. Not all his cases had epilepsy.

Since then, several authors have described examples of this syndrome with varying emphasis on the features mentioned previously. In practically all the reported series the syndrome appeared sporadically, and included epilepsy. Recently, Gilbert, McEntee, and Glaser (1963) have described four cases occurring in a family without epilepsy, under the title of 'familial myoclonus and ataxia'. A further four familial examples are presented here, together

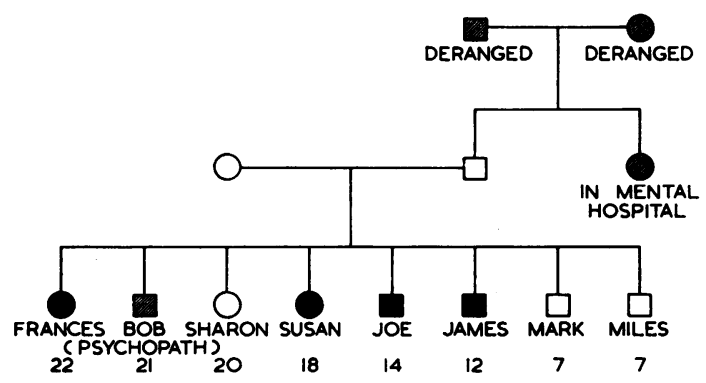

FIG. 1. Pedigree of the family described here. with the results of thalamotomy in the two most severely affected.

\section{CASE HISTORIES}

CASE 1 Frances D. is a 22-year-old woman of Polish $\stackrel{\mathbb{Q}}{\circ}$ extraction, who, at the age of 2 weeks, had had 'convul- $\$$ sions' for a brief period only. Difficulty with walking was $\overrightarrow{0}$ observed from infancy. Her parents observed that she was somewhat unsteady in her movements and tended to $\overrightarrow{\vec{\omega}}$ jerk her body forward spasmodically. Speech and mental $\omega_{\overparen{D}}$ development were moderately slow, but she progressed reasonably well, and was able to reach grade IX at school. Her unsteady gait, with the brief sudden jerking move- iv ments gradually progressed, until by the age of 16 she was unable to walk more than a few yards without assistances iv although she continued to feed herself and manage hẹ $N$ daily toilet.

On examination she was a thin, dark, young woman of low normal intelligence, and 'much distressed by her dis $\vec{c}$ ability. She had marked slurring dysarthria, as well as $\overparen{c}$ monotonous, nasal voice. There were bilateral signs cerebellar dysfunction with ataxia, intention tremor, dy $\vec{\theta}$ metria, and dyssynergia. In addition, there were frequesti myoclonic movements which involved the anterior trunk of muscles to a large extent, producing movements much like salaam spasms. Her head would bob forward with simultaneous powerful flexion of the abdominal musculature. Such movements interrupted her speech, and involved all limbs. The myoclonus appeared to be triggered $\frac{\mathscr{Q}}{D}$ by any effort to speak, emotional disturbances of minor $\cong$ degree, and by any attempt at a willed movement. When at $\overrightarrow{\vec{\sigma}}$ rest, and in sleep, the myoclonus ceased. There was no 3 tremor at rest, and no nystagmus. Power in her muscula- $\bar{F}$ ture was normal. A moderate symmetrical hypotonia was present. Deep reflexes were normal, and both plantar responses were flexor. Posterior column function was normal. General physical examination was within normalo limits.

Investigations included cerebrospinal fluid examination, blood and liver profiles, urine analysis, serum protein estimations, skull and chest radiographs, serum electrolytes, magnesium and copper levels, an electroence- $\mathrm{O}$ phalogram, and an air encephalogram. All were within normal limits.

While in hospital she was able to feed herself, and move about to a very limited degree, without assistance. $N$

On 8 July 1963 she underwent a. left thalamotomy. Immediately after the operation she developed a transient $n$ 
loss of speech and right limb weakness which cleared in three days. For the first three days all myoclonic movements ceased, but began to return by the end of a week, at first very slightly, then gradually increasing. There was, however, a distinctly lateralizing pattern. The right side was considerably quieter and her movements tended to flex her trunk laterally to the left as well as forward. There did not seem to be much improvement in the cerebellar signs, although subjectively the patient felt that she was improved. She was transferred to the Physical Restoration Centre in Regina where medical observers who had known her well thought that there was definite improvement. Unfortunately this observation was based entirely on objective clinical assessment and no claim for any scientific measurement of improvement is made.

Various drugs, including Artane, Cogentin, and Epanutin, were given before the thalamotomy and had no appreciable effect. Valium (Diazepam), in dosages reaching $30 \mathrm{mg}$. daily, produced some lessening of the frequency and range of the myoclonic movements. This was more apparent following thalamotomy.

CASE 2 Susan D. is the 16-year-old sister of the first patient. Her parents observed that she passed all her milestones at the appropriate times, and that mental and motor development were normal up to the age of 6 years. At this stage it was noticed that she was beginning the myoclonic movements which had been observed in Frances. At the same time, she was observed to be "clumsy and unbalanced'. Initially these signs were slight and hardly noticeable, but gradual progression became obvious over the years. The disability in this patient progressed at a faster rate when compared with her older sister, and by the age of 10 years she was chair-bound. Her mental achievements at school had been good and well up to average.

At the age of 14 years, coincident with the administration of oral polio vaccine, a marked deterioration was reported, and from this time onwards she was no longer able to move about unassisted and had to be fed and dressed.

On examination she was a young, well-developed girl of normal intelligence. As with her sister, her disability was a source of considerable despondency. Speech was severely dysarthric and constantly interrupted by explosive, widespread, myoclonic movements. These were of almost identical pattern as those of her older sister. She had, in addition, cerebellar signs of similar distribution but of greater severity. Any attempt at a willed movement was accompanied by several beats of myoclonus which completely disrupted the voluntary movement and rendered her speech quite unintelligible. All myoclonic movements ceased with rest and sleep. There was no tremor at rest, and no nystagmus. Power in her limbs and trunk was normal. Deep reflexes were present and both plantars were flexor. There was a moderate degree of symmetrical hypotonia. Posterior column function was normal. General physical examination was within normal limits.

Investigations were essentially similar to those done with the previous patient and all the results were within normal limits.

On 4 July 1963 she underwent a right-sided thalamotomy. For the first 24 hours all myoclonic movements ceased and for a time her speech was more intelligible, albeit still markedly dysarthric. The myoclonus returned within 36 hours, at first slight and clearly right-sided, but then gradually increasing in severity. The cerebellar signs improved slightly: again this was a clinical assessment. She had no post-operative difficulties and was transferred to the Physical Restoration Centre where it was reported by the observers who had previously handled her that there was a definite improvement, especially in the area of transferring from bed to wheel chair and back, and in her ability to maintain posture while sitting. She was now able to propel herself by wheel chair, an activity she was unable to perform before operation.

Of all the drugs exhibited, Diazepam appeared to have some beneficial influence on the frequency of the myoclonic movements.

CASE 3 Joseph D. is the 14-year-old brother of the two previously described patients. A normal pregnancy, fullterm normal delivery, and normal development up to the age of 3 years was reported. After an attack of 'flu' he developed a few infrequent 'convulsions', in which he would cry, hold his breath, roll his eyes upwards, especially when frustrated or injured. These episodes stopped spontaneously by the age of 5 or 6 years. From the age of 3 years his parents noticed a 'drunken gait' which at times seemed to improve. From this age very infrequent flexion spasms were observed, as in his sisters, involving the head, neck, and trunk. He was doing poorly at school and had difficulty with writing and reading; he did not take part in sport, mainly because of his excessive clumsiness.

On examination he was a tall, thin boy with a nasal, monotonous, and slurred speech.

He exhibited irregular, lateral jerking movements of his head which were exacerbated by anxiety or concentration. There was an irregular coarse tremor of myoclonic type mostly involving the left upper limb. Rapidly alternating movements of the upper limbs and the tongue were poorly performed. There was mild bilateral intention tremor. His gait appeared to be normal, but he was unable to hop on either foot. There was no nystagmus.

During the period of observation sudden truncal myoclonic movements were not seen. General physical examination and an electroencephalogram recorded at this time were within normal limits.

CASE 4 James D. is the 12-year-old brother of the above patients. Pregnancy, birth, and development up to the age of 2 years had been normal. At this time his alerted parents noticed occasional jerks of his head, and some 'lack of coordination' in his movements. A bout of whooping cough appeared to aggravate the abnormal movements. He continued to develop normally, and apart from his mild clumsiness and occasional head jerking, he has had no special disability. He was not keen to take part in sport at school but enjoyed learning. 
On examination he was a young boy of average physical and mental development. He had a dysarthric, slurred, monotonous voice, but the dysarthria was much less marked than in his siblings. He exhibited frequent, sudden, neck-flexing movements, especially during attention or on effort. Rapidly alternating movements in both upper limbs were poorly performed, and there was a very mild bilateral intention tremor. General physical examination and an electroencephalogram were within normal limits.

\section{SUMMARY OF FEATURES OF THE SYNDROME}

All affected members of the family exhibited cerebellar signs of varying degree, with dysarthria, intention tremor, and dyssynergia, but not nystagmus. In all cases the signs appeared to be bilateral, and first appeared in early infancy.

Myoclonus was present in all patients ranging from slight infrequent movements of the head and neck to severe flexion spasms involving all the ventral musculature and limb flexors. The disturbance was readily exacerbated by stimuli such as emotional excitement, concentration, and by efforts to move or speak.

Two members had 'seizures' of some sort for a brief period in very early life with subsequent cessation. In one patient, Joseph, it is possible that these were breath-holding spells. All members had normal electroencephalograms. Epilepsy did not appear to be a feature in these cases.

Mental development varied from normal to dull, without any evidence of a dementing process. There was, however, a strong history of psychiatric disorder in the family, but not in the known examples of the syndrome. One male sibling of 21 years exhibits psychopathic behaviour and at the time of writing is residing in a local correctional institute. A maternal aunt spent eight years in a mental hospital with a diagnosis of schizophrenia. The parents of the father were reported to be 'deranged' and apparently died in a mental hospital. Unfortunately, there is not a clear psychiatric history or diagnosis except in the case of the maternal aunt, and in the case of the 21-year-old sibling, Bob.

Although two of these cases had in the past been labelled Friedreich's ataxia, there was no clinical evidence to support this diagnosis. This is of some interest in view of the twin patients of Ramsay Hunt who had, in addition, Friedreich's ataxia and epilepsy.

Disability varied from slight to severe. In the two severely affected siblings there was a history of definite progression of the neurological disturbance.

A search of the literature appears to indicate that this is only the third recorded family with the disorder in this particular form. The others are recorded by Bradshaw (1954) and Gilbert et al. (1963). The으 mode of inheritance appears to be by an autosomal $\underset{\mathbb{}}{Z}$ dominant gene of incomplete penetrance.

\section{DISCUSSION}

Since the original description by Ramsay Hunt in 1914, Wohlfart and Höök (1951), Gilbert et al. S (1963), Aigner and Mulder (1960), Bradshaw (1954), Noad and Lance (1960), and Harriman and Millar. (1955) have described a variety of syndromes in- $\vec{F}$ cluding dyssynergia, myoclonus, and epilepsy. The $\stackrel{?}{+}$ existence of a variant of the Ramsay Hunt syndrome, i.e., progressive cerebellar dyssynergia, myoclonus, $\frac{\bar{\sigma}}{\bar{D}}$ and without epilepsy, was recognized by Ramsay $\frac{\pi}{\sigma}$ Hunt (1921), Wohlfart and Höök (1951), and Gilbert $\unrhd$ et al. (1963), and is unlike the disorder described by ${ }^{\circ}$ Unverricht (1891) and Lundborg (1903) because of $\vec{O}$ the absence of mental deterioration and epilepsy.

Myoclonus occurs in such a great variety of diseases $\vec{\omega}$ that any attempt at localizing the site of dysfunction $?$ is fraught with difficulty. Probably more than one $\bar{T}$ area may be involved. In 1930 Hodskins and Yakovlev published the results of a detailed investigation into 300 neurological patients in a state $\omega$ hospital. In 18 cases they also recorded myoclon $\sqrt{1}$ N and divided these into two groups: (1) myoclonie $\mathrm{N}$ epileptic cases and (2) myoclonic cases withooft epilepsy.

When attempting to localize the pathologic君 $\frac{5}{5}$ lesion they found that in those cases of myoclones ${ }^{D}$ without epilepsy the lesion was invariably present $\vec{b}$ the dentate nucleus but in some cases involved the inferior olives as well. In the epileptic cases, although the dentate nucleus was heavily involved, there was also severe involvement of the basal ganglia and of the midbrain, as well as more diffuse lesions. They inferred that the myoclonus was due to "cerebello- $\frac{}{\Phi}$ or dentato-tropic lesions'. Brock (1953) considers that the efferent cerebellar (dentate) rubral tract or the striatum may be involved.

Penfield and Kristiansen (1951) by means of electrophysiological experiments suggest that myoclonic seizures arise in the lower brain-stem and the spinal cord. Gastaut (1954), on the other hand, implicates the thalamus, as well as more caudally placed centres.

Denny-Brown (1962) found that when a large bilateral lesion of the nucleus ventralis lateralis oralis

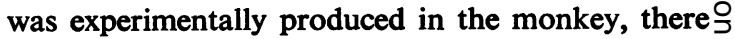
was a tendency for 'myoclonic flexions, as well as $>$ loss of tactile grasping, placing and contact righting'. $\mathrm{He}$ also comments on the work of Richter (1945) whon produced symmetrical necrosis of the globus pallidus in monkeys by exposure to carbon disulphide 0 gas with the development of myoclonic jerks as well 
as marked intention tremor. Myoclonus and epilepsy may also occur in Hallervorden-Spatz disease where the basal ganglia, the reticular zone of the substantia nigra, and the dentate nucleus may be involved by a fine powdery deposition of iron pigment, and by gliosis.

The ultimate neurological status of the two patients at present is only slightly improved and it remains to be seen if further surgical lesions of the nuclei of the thalamus will provide more improvement in this otherwise progressive and totally disabling condition. To a large extent the rationale for placing surgical lesions in the nuclei and the pathways of the extrapyramidal system is empirical, as it appears to be when the operations are performed for other conditions such as Parkinson's disease and intention tremor. Although this appears to be the first recorded instance of thalamotomy for dyssynergia cerebellaris myoclonica, we were guided by the considerable experience of others who placed lesions in the thalamus for movement disorders with benefit to the patient.

I am grateful to Dr. P. L. Steele and Dr. A. F. Huston for referring the patients, and to Dr. K. Kumar who performed the thalamotomies.

\section{SUMMARY}

Dyssynergia cerebellaris myoclonica was a term coined by Ramsay Hunt in 1921 to describe a rare disorder characterized by myoclonus, cerebellar dysfunction, with or without epilepsy.

Most of the published cases have been sporadic examples. The present series is the third recorded familial example of myoclonus, cerebellar dysfunction, and without epilepsy. The condition is progressive, arising in early childhood, and becomes physically disabling in time. It is to be differentiated from the rare familial disease of Unverricht and Lundborg by the absence of mental deterioration and epilepsy.

Various sites of possible dysfunction resulting in myoclonus and intention tremor are discussed. It is probable that more than one area is involved.

Thalamotomy was performed on the two oldest and most severely disabled of the four siblings of this family in an attempt to alleviate the severe intention tremor. Unexpectedly it was the myo- clonus and not the intention tremor which improved noticeably following the operations.

Of the various drugs exhibited, Diazepam appeared to be the only one which had any beneficial effect, both before and after the operations.

\section{REFERENCES}

Aigner, B. R., and Mulder, D. W. (1960). Myoclonus. Arch. Neurol. (Chic.) 2, 600-615.

Bradshaw, J. P. P. (1954). A study of myoclonus. Brain, 77, 138-157.

Brock, S. (1953). The Basis of Clinical Neurology, 3rd ed. Williams and Wilkins, Baltimore.

Denny-Brown, D. (1962). The Basal Ganglia. Oxford University Press, London.

Gastaut, H. (1954). The Epilepses. Electro-clinical Correlations. Charles C. Thomas, Springfield, Illinois.

Gilbert, G. J., McEntee, W. J., and Glaser, G. H. (1963). Familial myoclonus and ataxia. Pathophysiologic implications. Neurology (Minneap.), 13, 365-372.

Harriman, D. G. F., and Millar, J. H. D. (1955). Progressive familial myoclonic epilepsy in three families: its clinical features and pathological basis. Brain, 78, 325-349.

Hodskins, M. B., and Yakovlev, P. I. (1930). Anatomico-clinical observations on myoclonus in epileptics and on related symptom complexes. Amer. J. Psychiat., 9, 827-848.

Hunt, J. Ramsay (1921). Dyssynergia cerebellaris myoclonicaprimary atrophy of the dentate system; a contribution to the pathology and symptomatology of the cerebellum. Brain, 44, 490-538.

Lundborg, R. (1903). Die Progressive Myoklonus-Epilepie (Unverrichts Myoklonie), edited by Almquist and Wiksells, Uppsala.

Noad, K. B., and Lance, J. W. (1960). Familial myoclonic epilepsy and its association with cerebellar disturbance. Brain, 83, 618-630.

Penfield, W., and Kristiansen, K. (1951). Epileptic Seizure Patterns. Thomas, Springfield, Illinois.

Richter, R. (1945). 'Degeneration of the basal ganglia in monkeys from chronic carbon disulphide poisoning'. J. Neuropath. exp. Neurol., 4, 324-326.

Unverricht, H. (1891). Die Myoklonie, Franz Deuticke, Leipzig and Vienna.

Wohlfart, G., and Höök, O. (1951). A clinical analysis of myoclonus epilepsy (Unverricht-Lundborg), myoclonica cerebellar dyssynergy (Hunt) and heptato-lenticular degeneration (Wilson). Acta psychiat. scand., 26, 219-245.

\section{ADDENDUM}

One year later, in May 1964, both Susan and Frances had a further surgical lesion placed in the opposite ventrolateral nucleus of the thalamus. In both patients there was marked improvement in the range and frequency of the myoclonic movements. The most striking improvement was in the ability of both patients to sit unassisted. Intention tremor was smaller in range but appeared to be more rapid on the operated side. Speech remained monotonous and severely dysarthric.

However, the follow-up period thus far is measured in months, and it remains to be seen if the improvement is maintained. 